\title{
H 327 AO, HÍBRIDO TRILINEAL DE MAÍZ OLEOSO DE GRANO AMARILLO PARA REGIONES SUBTROPICALES DE MÉXICO
}

\section{H 327 AO, HIGH OIL YELLOW-KERNEL THREE-WAY MAIZE HYBRID ADAPTED TO SUBTROPICAL REGIONS OF MEXICO}

\author{
Ricardo E. Preciado-Ortiz ${ }^{1}$, Arturo D. Terrón-Ibarra', \\ Ma. Gricelda Vázquez-Carrillo² y Noel 0. Gómez-Montiel ${ }^{3}$
}

\begin{abstract}
'Instituto Nacional de Investigaciones Forestales, Agrícolas y Pecuarias (INIFAP), Programa de Mejoramiento Genético de Maíz, Campo Experimental Bajío, Celaya, Guanajuato. México. ${ }^{2}$ INIFAP, Laboratorio de Calidad de Maíz, Campo Experimental Valle de México, Texcoco, Estado de México, México. ${ }^{3}$ NIFAP, Programa de Mejoramiento Genético de Maíz, Campo Experimental Iguala, Iguala de la Independencia, Guerrero, México.
\end{abstract}

*Autor de correspondencia (preciado.ernesto@inifap.gob.mx); (repreciado@yahoo.com)

La producción de maíz de grano amarillo en México es deficitaria, durante 2016 se importaron 12.2 millones de t (FIRA, 2016) para satisfacer la demanda anual, que es superior a 15.3 millones de $t$, de las cuales $75.7 \%$ del maíz amarillo que se utiliza en México es de uso pecuario, principalmente como fuente de energía para la elaboración de alimentos balanceados. Partiendo de que el aceite del grano de maíz aporta 2.25 veces más energía que el almidón (Alexander, 1988), la utilización de maíz amarillo con alto contenido de aceite (ACA) podría tener impacto significativo en la alimentación pecuaria de rumiantes y monogástricos (bovinos, caprinos, porcinos, aves, entre otros). De acuerdo con Lambert (2000), un maíz ACA debe tener más del $6 \%$ de aceite total en el grano, mientras que un maíz normal tiene de 2.0 a $5.0 \%$.

El H 327 AO es un híbrido de grano amarillo oleoso (ACA), adaptado a regiones subtropicales, constituye una alternativa para elaborar alimentos balanceados en la industria pecuaria, así como para diversas industrias alimentarias de México; se forma por tres líneas denominadas Parental 1: LBDHAO1 (Línea Bajío Doble Haploide Amarilla Oleosa Uno), Parental 2: LBDHAO2, la genealogía de ambas líneas es PAN DH 142 y PAN DH 141, que son dos líneas doble haploides (LDH) derivadas de la Población Amarilla del Noroeste (PAN), ambas líneas forman el progenitor femenino de cruza simple (LBDHAO1 $\times$ LBDHAO2). El Parental 3: LBDHAO3, cuya genealogía es PAB DH 218, es una LDH derivada de la Población Amarilla del Bajío (PAB) y participa como progenitor masculino; el obtentor de las tres líneas parentales fue el INIFAP. EI germoplasma involucrado en las PAN y PAB fue descrito por Ortega-Corona et al. (2015) de la siguiente manera: la PAN incluye líneas, poblaciones y generaciones avanzadas de híbridos comerciales y experimentales, con adaptación al Noroeste y fue integrada en el Campo Experimental Norman Borlaug del INIFAP; la PAN se caracteriza por su grano de color anaranjado intenso y textura cristalina, además de tener tolerancia a roya y micoplasmosis. La PAB proviene de una población de amplia base genética de grano amarillo integrada en el Campo Experimental Bajío (CEBAJ) del INIFAP, con materiales básicos del Programa de Maíz, poblaciones, generaciones avanzadas y material nativo; la PAB es de grano amarillo dentado, ciclo de madurez y porte de planta intermedios. A partir de 2004 se inició la selección en PAN y PAB para ACA con un esquema de selección recurrente en el que se utilizaron familias de medios hermanos y se obtuvo un ciclo de selección por año En campo, se establecieron lotes aislados para recombinar las mejores 50 familias ACA del ciclo anterior. Se seleccionaron las cuatro mejores mazorcas dentro de cada familia, provenientes de plantas sanas, con competencia completa, sin acame y con altura de planta y mazorca que no excedieran la media de la población. En laboratorio, las semillas de cada una de las 200 mazorcas seleccionadas se analizó para ACA con un espectrofotómetro de rayos infrarrojos cercanos (NIRS) Infratec 1241 (Foss, Hilleroed, Dinamarca), donde se aplicó una presión de selección del $25 \%$; posteriormente, dentro de las 50 familias superiores se realizó la selección de semillas individuales con ACA a través del NIRS Infratec 1255 (Foss, Hilleroed, Dinamarca). La respuesta a la selección de estas poblaciones fue reportada por Preciado-Ortiz et al. (2013) y por OrtegaCorona et al. (2015).

Las LDH progenitoras del H 327 AO fueron derivadas a partir del octavo y séptimo ciclo de selección recurrente de la PAN y PAB. La generación de LDH se inició en el año 2011 a través de los siguientes pasos: 1) inducción de haploidía mediante la cruza de PAN y PAB con polen del inductor RWS $\times$ UH400, este último proporcionado por el Centro Internacional de Mejoramiento de Maíz y Trigo; 2) identificación de semilla haploide mediante el marcador Navajo R1-nj; 3) duplicación de haploides con colchicina 
como inhibidor mitótico y 4) autofecundación de plantas duplicadas para incrementar la semilla de las nuevas LDH (Prasanna, 2012). A partir del ciclo agrícola PV 2012 en el CEBAJ se realizó el incremento, selección per se y a través de cruzamientos de LDH.

El H 327 AO se caracteriza por su ACA, buen potencial de rendimiento, ciclo de madurez y porte intermedios, tolerancia al acame, espiga larga con alta presencia de ramas laterales, estigmas con antocianinas de intensidad tenue, buena cobertura y sanidad de mazorca, de tamaño medio, con 16 a 18 hileras de granos, ligeramente en espiral, grano color amarillo naranja, textura semicristalina y forma de la corona hendida. El progenitor femenino es de alto potencial de rendimiento (9 a $10 \mathrm{t} \mathrm{ha}^{-1}$ ), ciclo intermedio, porte intermedio, tamaño medio de mazorca, 16 a 18 hileras de granos, grano semidentado, de color amarillo y corona en forma convexa. El progenitor masculino es de ciclo intermedio, porte bajo, 14 a 16 hileras, grano amarillo cristalino, corona de forma convexa, espiga corta, abierta y alto número de ramas principales (Figura 1). El H 327 AO fue registrado en el Catálogo Nacional de Variedades Vegetales (CNVV) en el año 2018 ante el Servicio Nacional de Inspección y Certificación de Semillas (SNICS) con el número MAZ-1851-090318 y el Título de Obtentor con número de registro 2043.
El H 327 AO fue evaluado durante los años 2014, 2015 y 2016 en 13 ambientes de Guanajuato, Aguascalientes, Michoacán e Hidalgo, donde mostró un rendimiento de grano promedio de $9.5 \mathrm{t} \mathrm{ha}^{-1} \mathrm{y}$ un intervalo de 6.2 a $18.5 \mathrm{t} \mathrm{ha}$ 1 , intervalos de floración masculina de 66 a 82 y femenina de 68 a 84 d y alturas de planta de 170 a 320 cm y de mazorca de 68 a $165 \mathrm{~cm}$. En el Cuadro 1 se presentan las características físico-químicas del grano de H 327 AO en comparación con los testigos de grano amarillo y blanco DK2027Y y H 377

El H 327 AO presentó un índice de flotación de 33 \% que, de acuerdo con la Norma Mexicana, se clasifica como duro; en peso de 100 granos y peso hectolítrico se observaron valores menores que en los testigos; así mismo, se observó un mayor contenido de aceite, proteína y carotenos y un menor contenido de almidón que los testigos (VázquezCarrillo et al., 2018). Con lo anterior, se sustenta que el $\mathrm{H}$ 327 AO presenta características distintas a los testigos y representa una buena alternativa para aprovechamiento por la industria pecuaria y de transformación de México. La semilla básica del progenitor masculino (LBDHAO3) y la registrada del progenitor femenino (LBDHAO1 $\times$ LBDHAO2) está disponible, previa solicitud al INIFAP, para los interesados en la producción de semilla certificada del H $327 \mathrm{AO}$.

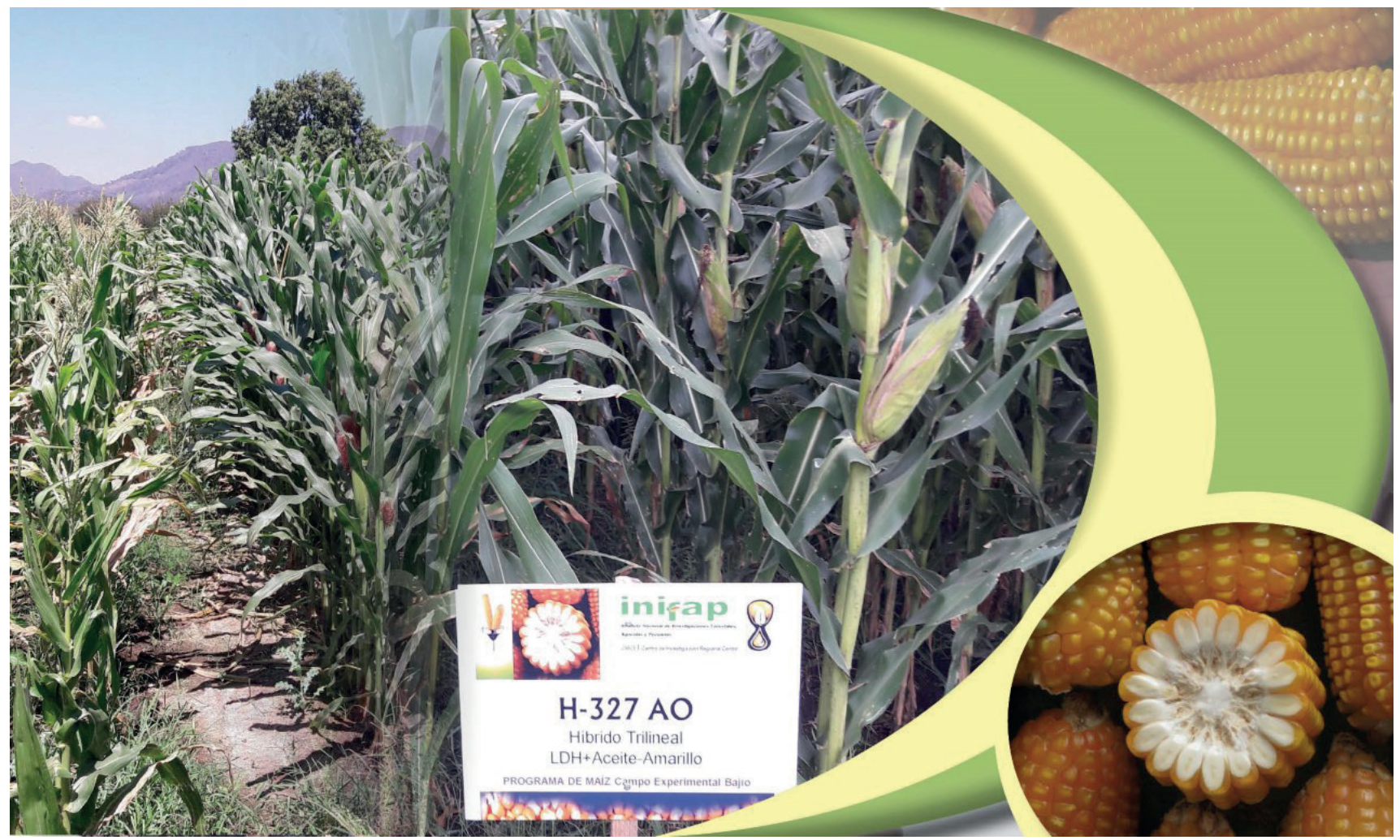

Figura 1. Aspecto de planta y mazorca del híbrido trilineal oleico amarillo H 327 AO. 
Cuadro 1. Medias de los caracteres de calidad de grano del híbrido trilineal H 327 AO y testigos comerciales a través de ocho ambientes de evaluación. PV 2014-2016.

\begin{tabular}{|c|c|c|c|c|c|c|c|}
\hline \multirow{2}{*}{ Genotipo } & \multirow{2}{*}{$\begin{array}{l}\text { Índice de } \\
\text { flotación } \\
(\%)\end{array}$} & \multirow{2}{*}{$\begin{array}{c}\text { Peso de } 100 \\
\text { granos } \\
\text { (g) }\end{array}$} & \multirow{2}{*}{$\begin{array}{c}\text { Peso } \\
\text { hectolítrico } \\
\left(\mathrm{kg} \mathrm{hL}^{-1}\right)\end{array}$} & $\begin{array}{c}\text { Contenido de } \\
\text { aceite }\end{array}$ & $\begin{array}{l}\text { Contenido } \\
\text { de almidón }\end{array}$ & $\begin{array}{c}\text { Contenido } \\
\text { de proteínas }\end{array}$ & \multirow{2}{*}{$\begin{array}{c}\text { Contenido de } \\
\text { carotenoides } \\
\qquad\left(\mu \mathrm{g} \mathrm{g}^{-1}\right)\end{array}$} \\
\hline & & & & \multicolumn{3}{|c|}{$(\%)$} & \\
\hline DK 2027 Y & $20.78 b$ & 38.68 a & $78.62 \mathrm{a}$ & $5.13 \mathrm{~b}$ & $69.03 \mathrm{a}$ & $9.15 \mathrm{c}$ & $2.52 b$ \\
\hline H 377 & $15.63 \mathrm{c}$ & $35.23 \mathrm{~b}$ & $78.51 \quad a$ & $5.07 \mathrm{~b}$ & $69.10 \mathrm{a}$ & $9.49 \mathrm{~b}$ & \\
\hline
\end{tabular}

Medias con letras diferentes son estadísticamente diferentes, (DMS, P $\leq 0.05)$. Los ambientes de evaluación fueron: Celaya, Gto. 2015; $7^{\text {a }}$ fecha; Celaya, Gto. 2015; 2ª fecha; Pabellón, Ags. 2015; Tarimbaro, Mich 2015; Celaya, Gto. 2016; $1^{\text {a }}$ fecha; Celaya, Gto. 2016; 2ª fecha; Tarimbaro, Mich 2016; y Juventino Rosas, Gto. 2016.

\section{BIBLIOGRAFÍA}

Alexander D. E. (1988) Breeding special nutritional and industrial types. In: Corn and Corn Improvement. G. F. Sprague and J. W. Dudley (eds.). 3rd edition. American Society of Agronomy. Madison, Wisconsin. pp:869-880.

FIRA, Fideicomisos Instituidos en Relación con la Agricultura (2016) Panorama Agroalimentario. Maíz 2016. Dirección de Investigación y Evaluación Económica y Sectorial. Fideicomisos Instituidos en Relación con la Agricultura, Banco de México. México, D. F. 40 p.

Lambert J. R. (2000) High-oil corn hybrids. In: Specialty Corns. A. R. Hallauer (ed.). 2nd edition. CRC Press. Boca Raton, Florida. pp:137-154.

Ortega-Corona A., R. Picón-Rico, R. E. Preciado-Ortiz, A. D. Terrón-Ibarra, M. J. Guerrero-Herrera, S. García-Lara and S. O. Serna-Saldivar (2015)
Selection response for oil content and agronomic performance in four subtropical maize populations. Maydica 60:1-8.

Prasanna B. M. (2012) Doubled haploid (DH) technology in maize breeding: an overview. In: Doubled Haploid Technology in Maize Breeding: Theory and Practice. B. M. Prasanna, V. Chaikam and G. Mahuku (eds). CIMMYT. México, D. F. pp:1-8.

Preciado-Ortiz R. E., S. García-Lara, S. Ortiz-Islas, A. Ortega-Corona and S. 0. Serna-Saldivar (2013) Response of recurrent selection on yield, kernel oil content and fatty acid composition of subtropical maize populations. Field Crops Research 142:2735, https://doi.org/10.1016/j.fcr.2012.11.019

Vázquez-Carrillo M. G., R. E. Preciado-Ortíz, D. Santiago-Ramos, N. PalaciosRojas, A. Terrón-Ibarra y A. Hernández-Calette (2018) Estabilidad del rendimiento y calidad de grano y tortilla de nuevos híbridos de maíz con valor agregado para el subtrópico de México. Revista Fitotecnia Mexicana 41:509-518. 